\title{
Cloud Tracking in METEOSAT Images Using GIMP
} Program

\author{
Amelia Carolina Sparavigna 1 \\ ${ }^{1}$ Department of Applied Science and Technology, Politecnico di Torino, Italy
}

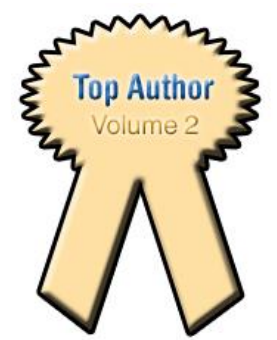

Abstract: Cloud tracking is the analysis of remote sensing images from which it is possible to evaluate the speed of winds drifting the clouds. Here we are proposing a simple approach to the cloud drift, based on a method that uses GIMP, the GNU Image Manipulation Program, applied to METEOSAT images.

Keywords: Satellite images, Remote sensing, Image processing, GIMP.

\section{Introduction}

In some recent papers [1,2], we have used the images of Google Earth and a processing tool, the GNU Image Manipulation Program (GIMP), to study the motion of sand dunes. Actually, the evaluation of migration rates of dunes is requiring large length and time scales; however, if some time series of satellite images, recorded for a given dune field, are available, the measurements can be easily made using GIMP [1]. Here we will see that we can apply a similar approach to the cloud tracking too.

Cloud tracking is the analysis of time series of images given by geostationary satellites, from which it is possible to evaluate the speed of winds drifting the clouds. Essentially, the drift is given by the displacement of clouds, which is measured using the known distances of some landmarks in the image frame, and by the time elapsed between the recorded images.

Several researches on cloud tracking had been performed in the past, but their number is still growing steadily. Besides the aim of improving or developing new algorithms, a reason for this growth is in an increasing number of countries launching satellites with a consequent large amount of data to analyze. Today, there are several organizations, besides the National Oceanic and Atmospheric Administration (NOAA), the National Aeronautics and Space Administration (NASA) and the European Organisation for the Exploitation of Meteorological Satellites (EUMETSAT), that have gained access to worldwide satellite data [3]. Acquired from geostationary and polar orbiting satellites, these data allow determining the behavior of weather patterns and their global nature [3].

\section{Satellite monitoring}

Weather monitoring was one of the first civilian applications of satellite remote sensing. The first weather satellite was TIROS-1, launched in 1960 by the United States. Several other weather satellites had been launched in the next five years, positioned in near-polar orbits, to give a coverage of global weather patterns. In 1966, NASA launched the geostationary ATS-1, giving images of the Earth's surface and clouds every half hour. For the first time, the development and movement of weather systems were routinely monitored [4].

Geostationary satellites can measure the winds because we can observe the movement of some features in the images they are recording. In the visible range, during the day, these features are the clouds. The distance traveled by a cloud divided by the time interval between images is giving the speed by which the cloud is drifted. The use of images in the infrared range allows the determination of the cloud height and night-time tracking too [5]. Besides the clouds, there are some other features from remote sensing, such as those available from water vapor imagery, which can be automatically tracked with a computer; this tracking is able to give information also in the case of absence of clouds [5].

The geostationary satellite GOES system followed the ATS series. These satellites, designed by NASA for NOAA, were able to record frequent small-scale imaging of the Earth's surface and cloud cover. The satellites were equipped to have images from several channels, which were sensing visible and infrared reflected and emitted solar radiation [6]. It is important to remark that infrared capability allows for night imaging too. As told in [6], the visible band in the wavelength range $0.52-0.72$ microns can see cloud, pollution and haze; if shortwave infrared is used, it can see fog at night, but also discriminate water clouds and snow or ice clouds during daytime. The shortwave IR can also detect fires and, nighttime, the temperature of the sea surface. The specific features of the other bands can be find at [6]. 


\section{The methods of tracking}

The method of using the satellites to see motions in atmosphere is coming from the pioneering works of Tetsuya Fujita [7]. At the beginning of the 1960s, when meteorological satellites were introduced, he developed techniques for measurements using sequences of images from polar orbiting platforms first and then from the geostationary platforms [7]. Fujita's works related the track of clouds to the flow patterns in the atmosphere. His results were soon transferred into routine operations, suitable for the national forecast centers. Then, the cloud motion vectors, which can be obtained from geostationary satellite imagery, became an important data source of meteorological information [7]. Of course, there is a large number of publications on cloud tracking starting from those of Fujita, and the analysis of all these publications would require a specific work. Let us just note a fact which is emerging from the titles of these publications: with the satellites orbiting near other planets of the solar system, cloud-tracking wind vectors and temperature fields analysis can been applied to Venus and Mars, to understand their atmospheric behavior and compare to that of Earth $[8,9]$.

\section{Using GIMP}

Let us suppose of studying the cloud drift, with an approach that could be used by undergraduate students too, simple but at the same time able of stimulating their interest for environmental issues. The method that we can use is based on METEOSAT images and GIMP. Let us suppose of having two images of the same area, recorded by a geostationary satellite at two different times, for instance A1 and A2 in the Figure 1 (infrared images, Atlantic Ocean, Canaries). If we are able to compare the positions of the clouds and their displacements on the given time interval, we have the speed of the cloud drift. For the comparison, we can follow this way. A1 is modified with the GIMP Invert command that inverts all the pixel colors and brightness values: the image $\mathrm{A} 3$ is obtained. After, on A3, the "posterize" tool is applied. This tool is designed to weigh the pixel colors of the image and reduce the number of colors, while maintaining a semblance of the original image characteristics. Posterize is activated from the image menu through Tools > Color Tools > Posterize or Colors > Posterize. Let us posterize A3 in black and white: we obtain A4. For the image A2, we use "posterize" on the direct image, obtaining A5. Then, A4 and A5 are combined, superimposing them in the same image A6, using the commands Windows >
Dockable Dialogs, from the GIMP image menu. In this image, we see the old position of clouds in black, the new position in white. Since the time interval is known, once we measure the distances, we have the cloud drift.

To measure the distance, we use another instrument of GIMP. In the panel of tools, we can select the pair of compasses. Using it, below the image, we have the distance, in pixels, and the angle in degrees, given by this instrument. In the Figure 2, lower panel, we show four distances, in pixels, measured by the pair of compasses. However, to have the distances in $\mathrm{km}$, we need to know at least a distances between two landmarks in the image frame: we can have it from Google Earth, as shown in the example proposed in the upper panel of Figure 2. In this panel, the chosen distance is 89 pix, equal to $63 \mathrm{~km}$. In the lower panel, this distance is equivalent to 20 pixels. It easy to apply the proportion and have the distances in $\mathrm{km}$, traveled by the clouds. In Figure 2, we have marked the drift of three clouds and evaluated the speed of it.

As previously told, the subject of this paper is not the improvement of cloud tracking methods, but it is that of proposing an approach simple enough to be used by undergraduate students, aiming to stimulate their interest for environmental issues and for studies on remote sensing and image processing.

\section{References}

[1] A.C. Sparavigna (2013). The GNU Image Manipulation Program applied to study the sand dunes, International Journal of Sciences, Volume 2, Issue 9, Pages 1-8.

[2] A.C. Sparavigna (2013). A case study of moving sand dunes: The barchans of the Kharga Oasis, International Journal of Sciences, Volume 2, Issue 8, Pages 95-97.

[3] R.A. Kohrs, M.A. Lazzara, J.O. Robaidek, D.A. Santek and S.L. Knuth (2014). Global satellite composites - 20 years of evolution, Atmospheric Research, Volumes 135-136, Issue January, Pages 834.

[4] M. Williamson (2006). Spacecraft technology: The early years, IET, ISBN 978-0-86341-553-1.

[5] Vv. Aa. (2014). How do I read the cloud drift wind data? NOAA National Climatic Data center, www.ncdc.noaa.gov/oa/ $\mathrm{rsad} / \mathrm{satfaq} /$ cloud-drift-wind-read.html

[6] Vv. Aa. (2014). Weather satellites/sensors, Natural Resources Canada, Government of Canada, www.nrcan.gc.ca/ earth-sciences/ geomatics/ satellite-imagery-air-photos/ satellite-imageryproducts/ educational-resources/9387

[7] W.P. Menzel (2001). Cloud tracking with satellite imagery: From the pioneering work of Ted Fujita to the present, Bull. Amer. Meteor. Soc., Volume 82, Issue 1, Pages 33-47.

[8] C.F. Wilson and A. Piccialli (2012). Characterizing atmospheric waves on Venus, Earth, and Mars, Eos Trans. American Geophysical Union, Volume 93, Issue 23, Page 220.

[9] R. Francis, J. Moores, K. McIsaac, D. Choi and G. Osinski (2014). Observations of wind direction by automated analysis of images from Mars and the MSL rover, Acta Astronautica, Volume 94, Issue 2, Pages 776-783. 

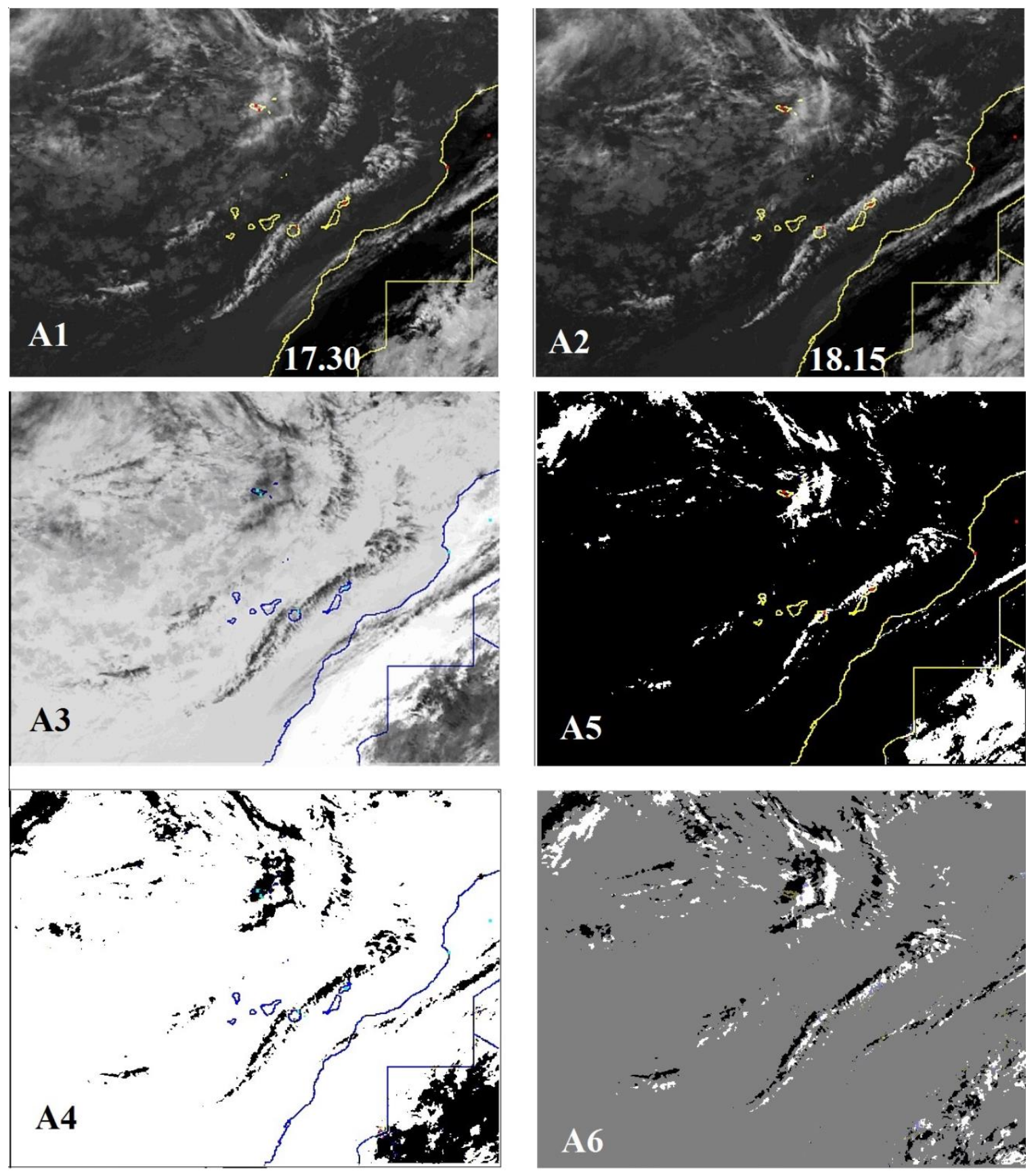

Figure 1: Let us use two infrared remote sensing images, acquired on the same day at different hours (A1 at 17.30 and A2 at 18.15, Atlantic Ocean, Canaries, Courtesy: EUMETSAT and SAT24). A1 is modified with GIMP Invert

command that inverts all the pixel colors and brightness values: the image A3 is obtained. After, on A3, the "posterize" tool is applied. This tool is designed to weigh the pixel colors of the image and reduce the number of colors, while maintaining a semblance of the original image characteristics. Posterize is activated from the image menu through Tools > Color Tools > Posterize or Colors > Posterize. Let us posterize A3 in black and white: we obtain A4. For the image A2, we use "posterize" on the original image, obtaining A5. Then, A4 and A5 are superimposed in the same image A6, using the commands Windows > Dockable Dialogs. In A6, we see the old positions of clouds in black, the new positions in white. Since the time interval is known, measuring the displacement distances and dividing them by the time interval we can have the speed of the cloud drift. 

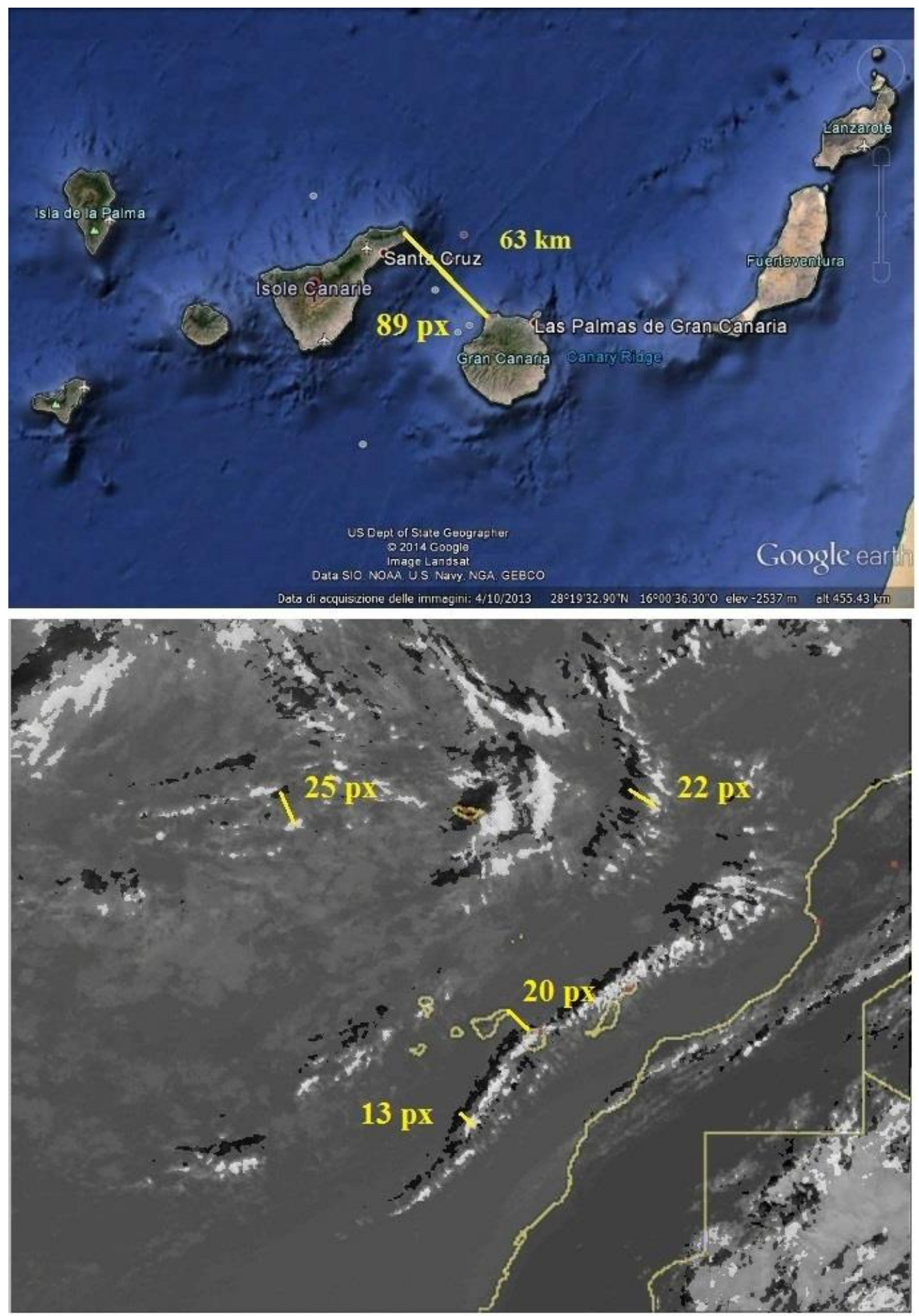

Figure 2: To measure the distances, we use another instrument of GIMP. In the panel of tools, we can select the pair of compasses, by which we can measure the distances (in pixels) of two points of the image. In the lower panel, we show four distances, in pixels, measured in such manner. However, to have the distances in $\mathrm{km}$, we need at least a distance between two landmarks visible in the image, for instance that of $20 \mathrm{px}$, in $\mathrm{km}$. We have it from Google Earth, as shown in the upper panel: the distance is $63 \mathrm{~km}$, equal to 89 pixels. It easy to apply the proportion and have the cloud drift in $\mathrm{km}$. In the image, three clouds are considered, with displacements of $8.5 \mathrm{~km}, 15.5 \mathrm{~km}$ and $17.7 \mathrm{~km}$. Speeds are $11 \mathrm{~km} / \mathrm{h}, 21 \mathrm{~km} / \mathrm{h}$ and $24 \mathrm{~km} / \mathrm{h}$, approximately. (Courtesy: EUMETSAT, SAT2 4 and

Google Earth). 